\title{
Caracterização física e hidráulica de solos em bacias experimentais do semiárido brasileiro, sob manejo conservacionista ${ }^{1}$
}

\author{
José Roberto L. da Silva², Abelardo A. A. Montenegro² \& Thais E. M. dos Santos ${ }^{2}$
}

\begin{abstract}
RESU M O
Para estudos de movimento da água no solo tornam-se imprescindíveis o conhecimento de suas propriedades físicas e hidráulicas e suas correlações. Ambas as propriedades têm importância fundamental no armazenamento e transporte de água, tal como, também, de nutrientes e no controle da infiltração, sendo influenciadas pelas condições de superfície do solo. N este contexto buscou-se realizar, com o presente estudo, a caracterização físico-hídrica de solos de duas bacias experimentais (Jatobá e Mimoso/Pesqueira, PE) do semiárido brasileiro, em diferentes profundidades e distintas coberturas vegetais (cobertura natural, solo descoberto, cultivo morro abaixo, palma em curva de nível e barramento de pedra com cobertura morta). O bservou-se alta variabilidade da condutividade hidráulica nas camadas até $50 \mathrm{~cm}$ além de decaimento dos teores de areia, de matéria orgânica e da condutividade hidráulica com a profundidade, no perfil dos solos, nas bacias estudadas. Já com relação às diferentes coberturas vegetais constatou-se que não houve diferença significativa entre médias da condutividade hidráulica entre os diferentes tratamentos conservacionistas avaliados, para as profundidades estudadas.
\end{abstract}

Palavras-chave: condutividade hidráulica, curva de retenção de umidade, permeâmetro de Guelph, cobertura vegetal

\section{Hydraulic and physical characterization of soils in experimental basins of the brazilian semiarid under conservation management}

\begin{abstract}
For the study involving the water movement in soil, it becomes imperative to know their physical and hydraulic properties and their correlations. Both are of fundamental importance in the storage and transport of water and nutrients and in controlling infiltration, being influenced by the conditions of the soil surface. In this context, this study aimed to perform physical and hydraulic characterization of the soil in two experimental basins (Jatoba and Mimoso/Pesqueira, PE), in the brazilian semiarid, at different depths and vegetation cover conditions (natural vegetation, bare soil, cultivation downhill, palm contour and stone micro walls with mulching). High variability of hydraulic conductivity was observed in layers up to $50 \mathrm{~cm}$. Besides this there was a decrease in sand content, organic matter, and hydraulic condutivity with depth, the soil profile in the studied basins. On the other hand, with respect to different vegetation cover, it was observed that there was no significant difference in mean hydraulic conductivity among the different evaluated conservation treatments, for the analysed depths.
\end{abstract}

Key words: hydraulic conductivity, moisture retention curve, Guelph permeameter, vegetation cover

1 Parte da Dissertação de Mestrado do primeiro autor apresentada ao PPG em Engenharia Agrícola, U FRPE.

${ }^{2}$ DTR /U FRPE, Rua D om Manoel de Medeiros s/n, Dois Irmãos, CEP 52171-900 Recife, PE. Fone: (81) 3320-6264 E-mail: rlopes.s@gmail.com; abelardo.montenegro@yahoo.com.br; thaisemanuelle@hotmail.com 


\section{INTRODUÇÃO}

Estudos relacionados às características físico-hídricas de solos em bacias do semiárido têm sido desenvolvidos por Grupos de Pesquisa inseridos na Rede de Hidrologia do Semiárido (REHISA), cujo objetivo principal é ordenar e ampliar o conhecimento sobre o comportamento hidrológico de bacias representativas do semiárido brasileiro.

As propriedades físicas e hidráulicas do solo influenciam nos processos hidrológicos, incluindo a infiltração, a erosão, a redistribuição de umidade e o transporte de solutos através do perfil. Bagarello et al. (2010) ressaltam que a condutividade hidráulica, a densidade do solo e sua umidade, são as variáveis mais significativas a serem monitoradas em uma bacia hidrográfica. Em bacia do semiárido brasileiro Souza et al. (2008) verificaram que a condutividade hidráulica e a curva de retenção de água no solo apresentaram elevada variabilidade espacial relacionadas, portanto, ao manejo agrícola tal como a outros atributos físicos.

A condutividade hidráulica pode ser estimada em laboratório ou em campo tendo, em laboratório, o inconveniente de se utilizar amostras muitas vezes de pouca representatividade, devido às dimensões reduzidas e ao risco de dano na sua estrutura. Carvalho et al. (2007) ressaltam que a determinação da condutividade hidráulica em campo apresenta maior precisão quando comparada com a de laboratório. Dentre os métodos de campo pode-se destacar o baseado em simuladores de chuva, que possibilita a estimativa da taxa de infiltração em escala não pontual, e no permeâmetro de Guelph, que permite quantificar localmente a taxa de infiltração, a condutividade hidráulica, o potencial de fluxo mátrico e a sortividade, com relativa rapidez e precisão (Reynolds \& Elrick, 1985). Montenegro \& Montenegro (2004) comentam que o permeâmetro de Guelph possui várias vantagens em relação a outros métodos, dentre elas a fácil montagem em campo, podendo-se investigar, de forma rápida, o comportamento hidráulico de perfis do solo. Nos últimos anos diversos autores vêm utilizando o permeâmetro de Guelph. Assis \& Lanças (2005) investigaram a relação entre a condutividade hidráulica, estimada pelo permeâmetro de Guelph, com os atributos físicos do solo submetidos a diferentes manejos. Adicionalmente, Eguchi et al. (2003) utilizaram o permeâmetro de Guelph para estudo de variabilidade espacial da condutividade hidráulica do solo saturado e da taxa de infiltração básica in situ.

Dentre as variáveis que interferem no comportamento hidráulico dos solos, merecem destaque as condições de superfície e subsuperfície, relacionadas a técnicas conservacionistas. Silva et al. (2005) ressaltam que a utilização de sistemas conservacionistas afeta o armazenamento de água no solo e tende a aumentar a condutividade hidráulica, próximo à superfície do solo. Além do manejo, a composição granulométrica e o teor de matéria orgânica também influenciam na retenção de água e na condutividade hidráulica. Utilizando simulador de chuva tipo pendular, Santos et al. (2008) constataram a influência da cobertura do solo e tratamentos conservacionistas na taxa de infiltração do solo, em Neossolo Flúvico na Bacia do Riacho Mimoso, Pesqueira, PE.

Assim, o presente trabalho teve por objetivo realizar uma caracterização físico-hídrica mais detalhada de perfis de solos predominantes na Bacia Representativa do Alto Ipanema, no semiárido de Pernambuco, a fim de ampliar o conhecimento do potencial hidrológico de bacias hidrográficas experimentais dessas regiões e gerar um banco de dados para subsidiar a modelagem hidrológica nas bacias estudadas, verificando também a influência de tratamentos conservacionistas na condutividade hidráulica em subsuperfície.

\section{Material e MÉTOdos}

O estudo foi desenvolvido nas bacias do Jatobá e Mimoso, entre as coordenadas (UTM) 715000 e 9065000,735000 e 9065000 (Datum WGS84). Tais bacias estão localizadas no município de Pesqueira e inseridas na Bacia Representativa do Alto Ipanema, no semiárido de Pernambuco, ou seja, uma das sub-bacias investigadas pelo Grupo de Pesquisa em Recursos Hídricos (GPRH) da Universidade Federal Rural de Pernambuco que compõem a Rede de Hidrologia do Semiárido (REHISA). A Figura 1 apresenta o mapa de locação da área de estudo.

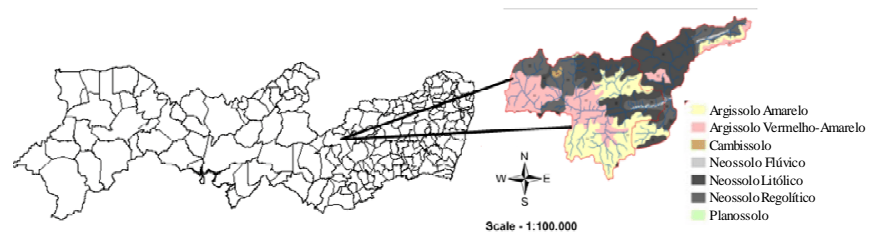

Figura 1. Localização da área de estudo apresentando a delimitação da Bacia Representativa do Alto Ipanema, com destaque para a rede de drenagem e os tipos de solo

De acordo com a classificação de Köppen, o clima na região é do tipo BSs'h' (extremamente quente, semiárido), com precipitação total anual média de $730 \mathrm{~mm}$ e evapotranspiração potencial anual média de $1683 \mathrm{~mm}$ (Montenegro \& Montenegro, 2004).

As coletas das amostras de solo deformadas e indeformadas foram realizadas no período de janeiro a fevereiro de 2009 e caracterizadas no Laboratório de Física do Solo da Universidade Federal Rural de Pernambuco (UFRPE), incluindo determinações da densidade global, densidade das partículas, porosidade e composição textural, utilizando-se o método do densímetro (EMBRAPA, 1997).

Classificou-se a variabilidade das propriedades físicas com base nos valores de coeficiente de variação (C.V), segundo Warrick \& Nielsen (1980), como baixa (C.V < 12\%), média (12 C.V $\leq 62 \%$ ) e alta (C.V > 62\%).

As curvas de retenção de água e umidade do solo foram avaliadas em extrator de Richards, no Laboratório de Física do Solo da Universidade Federal de Viçosa (UFV), conforme EMBRAPA (1997), com as umidades sendo estimadas nas tensões de 0,$01 ; 0,033 ; 0,1 ; 0,5$ e 1,5 MPa, a partir de amostras 
indeformadas; posteriormente, as curvas foram ajustadas ao modelo proposto por van Genuchten (1980) (Eq. 1):

$$
\theta=\theta_{\mathrm{r}}+\frac{\theta_{\mathrm{s}}-\theta_{\mathrm{r}}}{\left[1+\left[\alpha_{\mathrm{vG}}\left|\Psi_{\mathrm{m}}\right|\right]^{\mathrm{n}}\right]^{1-\frac{1}{\mathrm{n}}}}
$$

em que:

n - é um parâmetro de ajuste aos dados experimentais

$\theta$ - umidade atual, $\mathrm{LL}^{-3}$

$\theta_{\mathrm{r}}$ - umidade residual, $\mathrm{LL}^{-3}$

$\theta_{\mathrm{s}}$ - umidade de saturação, $\mathrm{L} \mathrm{L}^{-3}$

$\Psi_{\mathrm{m}}$ - potencial matricial, $\mathrm{kPa}$

$\alpha_{\mathrm{vG}}$ - parâmetro alfa da função de van Genutchen (1980), $\mathrm{kPa}^{-1}$

Em campo, a condutividade hidráulica foi determinada através de um permeâmetro de carga constante, tipo Guelph, cujo dispositivo permite a estimativa do parâmetro alfa $\left(\alpha_{\mathrm{G}}\right)$ da função $\mathrm{K}\left(\psi_{\mathrm{m}}\right)$ proposta por Gardner (1958) (Eq. 2), a partir da medição da condutividade hidráulica do solo saturado $\left(\mathrm{K}_{0}\right)$ de campo e do potencial de fluxo mátrico $\left(\phi_{\mathrm{m}}\right)$ :

$$
\mathrm{K}\left(\psi_{\mathrm{m}}\right)=\mathrm{K}_{0} \mathrm{e}^{\alpha_{\mathrm{G}} \psi_{\mathrm{m}}}
$$

em que:

$\alpha_{\mathrm{G}}$ - parâmetro alfa da função de Gardner, podendo o mesmo ser determinado pela Eq. 3 abaixo:

$$
\alpha_{\mathrm{G}}=\frac{\mathrm{K}_{0}}{\phi_{\mathrm{m}}}
$$

Calcularam-se os valores de condutividade hidráulica do solo saturado $\left(\mathrm{K}_{0}\right)$ segundo Reynolds \& Elrick (1986), como recomendado por Soil Moisture (1991) (Eqs. 4 a 8). Nesta investigação foram adotadas quatro cargas hidráulicas (2,5; 5,$0 ; 7,5$ e 10,0 cm), o que permitiu utilizar-se seis pares de cargas hidráulicas diferentes nas estimativas, sendo cada par de lâminas usadas considerado repetição na estimativa dos parâmetros.

$$
\mathrm{K}_{0}=\mathrm{G}_{2} \mathrm{Q}_{2}-\mathrm{G}_{1} \mathrm{Q}_{1}
$$

em que:

$$
\begin{gathered}
\mathrm{G}_{1}=\frac{\mathrm{H}_{2} \mathrm{C}_{1}}{\pi\left(2 \mathrm{H}_{1} \mathrm{H}_{2}\left(\mathrm{H}_{2}-\mathrm{H}_{1}\right)+\mathrm{a}^{2}\left(\mathrm{H}_{1} \mathrm{C}_{2}-\mathrm{H}_{2} \mathrm{C}_{1}\right)\right)} \\
\mathrm{G}_{2}=\frac{\mathrm{H}_{1} \mathrm{C}_{2}}{\pi\left(2 \mathrm{H}_{1} \mathrm{H}_{2}\left(\mathrm{H}_{2}-\mathrm{H}_{1}\right)+\mathrm{a}^{2}\left(\mathrm{H}_{1} \mathrm{C}_{2}-\mathrm{H}_{2} \mathrm{C}_{1}\right)\right)} \\
\mathrm{Q}_{1}=\mathrm{XR}_{1}
\end{gathered}
$$

$$
\mathrm{Q}_{2}=\mathrm{XR}_{2}
$$

no qual:

$\mathrm{X}$ - área do reservatório, $\mathrm{L}^{2}$

$\mathrm{H}_{1}$ e $\mathrm{H}_{2}$ - cargas hidráulicas aplicadas, L; $\mathrm{C}_{1}$

$\mathrm{C}_{2}$ - fatores de forma dependentes da relação $\mathrm{H}_{1} / \mathrm{a}$ e $\mathrm{H}_{2} / \mathrm{a}$

$\mathrm{R}_{1}$ e $\mathrm{R}_{2}$ - taxas de variação de carga estacionárias, correspondentes às cargas hidráulicas, LT $^{-1}$

a - raio do furo de sondagem, $\mathrm{L}$

Já o fluxo do potencial mátrico $\left(\phi_{\mathrm{m}}\right)\left(\mathrm{L}^{2} \mathrm{~T}^{-1}\right)$ foi determinado pelas Eqs. 9 a 11, conforme Soil Moisture (1991).

$$
\phi_{\mathrm{m}}=\mathrm{G}_{3} \mathrm{Q}_{1}-\mathrm{G}_{4} \mathrm{Q}_{2}
$$

em que:

$$
\begin{aligned}
& \mathrm{G}_{3}=\frac{\left(2 \mathrm{H}_{2}^{2}+\mathrm{a}^{2} \mathrm{C}_{2}\right) \mathrm{C}_{1}}{2 \pi\left(2 \mathrm{H}_{1} \mathrm{H}_{2}\left(\mathrm{H}_{2}-\mathrm{H}_{1}\right)+\mathrm{a}^{2}\left(\mathrm{H}_{1} \mathrm{C}_{2}-\mathrm{H}_{2} \mathrm{C}_{1}\right)\right)} \\
& \mathrm{G}_{4}=\frac{\left(2 \mathrm{H}_{1}^{2}+\mathrm{a}^{2} \mathrm{C}_{1}\right) \mathrm{C}_{2}}{2 \pi\left(2 \mathrm{H}_{1} \mathrm{H}_{2}\left(\mathrm{H}_{2}-\mathrm{H}_{1}\right)+\mathrm{a}^{2}\left(\mathrm{H}_{1} \mathrm{C}_{2}-\mathrm{H}_{2} \mathrm{C}_{1}\right)\right)}
\end{aligned}
$$

Segundo Zhang et al. (1998), os fatores de forma C dependem não apenas da relação H/a mas também da textura do solo. Para as três principais classes de solo (areia, argila estruturada e argila desestruturada), esses autores sugerem, respectivamente, as Eqs. 12 a 14, expressas como:

$$
\begin{aligned}
& \mathrm{C}=\left(\frac{\mathrm{H} / \mathrm{a}}{2,074+0,093 \mathrm{H} / \mathrm{a}}\right)^{0,754} \\
& \mathrm{C}=\left(\frac{\mathrm{H} / \mathrm{a}}{1,992+0,091 \mathrm{H} / \mathrm{a}}\right)^{0,683} \\
& \mathrm{C}=\left(\frac{\mathrm{H} / \mathrm{a}}{2,102+0,118 \mathrm{H} / \mathrm{a}}\right)^{0,655}
\end{aligned}
$$

A Eq. (12) é usada para condições em que o valor de $\alpha_{\mathrm{G}}$ é $\geq$ $0,12 \mathrm{~cm}^{-1}$; a Eq. (13), para $\alpha_{\mathrm{G}}=0,04 \mathrm{~cm}^{-1}$ e a Eq. (14), para $\alpha_{\mathrm{G}}=$ $0,01 \mathrm{~cm}^{-1}$. Alternativamente, o parâmetro $\alpha_{\mathrm{G}}$ pode ser estimado a partir das características edáficas, conforme a Tabela 1, segundo Elrick et al. (1989).

Com base na Tabela 1 , os solos do presente estudo apresentam valor de $\alpha_{\mathrm{G}}$ de $0,12 \mathrm{~cm}^{-1}$, sendo utilizada, nessas condições, a Eq. (12) para o cálculo do fator de forma.

Durante a realização dos testes valores negativos da condutividade hidráulica do solo saturado foram encontrados a partir da Eq. 4; tais valores ocorrem, geralmente, em 
Tabela 1. Categorias de estrutura e textura do solo e val ores de referência do parâmetro $\alpha_{G}{ }^{*}$

\begin{tabular}{|c|c|}
\hline Categoria de estrutura e textura do solo & $\alpha_{G}\left(\mathrm{~cm}^{-1}\right)$ \\
\hline $\begin{array}{l}\text { Material compactado, sem estrutura, textura argilosa ou siltosa como material usado em fundo de aterros, lacustre ou sedimentos } \\
\text { marinhos }\end{array}$ & 0,01 \\
\hline Solos de textura fina (argilosos ou siltosos) e não estruturados; pode-se incluir também algumas areias finas & 0,04 \\
\hline $\begin{array}{l}\text { A maioria dos solos estruturados a partir de argilas também inclui solos de estrutura média e areia fina. A categoria mais aplicável } \\
\text { para solos agrícolas }\end{array}$ & 0,12 \\
\hline $\begin{array}{l}\text { Areias grossas e cascalho; também se pode incluir alguns solos altamente estruturados, com grandes números de rachaduras e/ou } \\
\text { macroporos }\end{array}$ & 0,36 \\
\hline
\end{tabular}

condições de solos heterogêneos, casos em que se recomenda a utilização de uma carga hidráulica para validação dos resultados da condutividade hidráulica do solo saturado $\left(\mathrm{K}_{0}\right)$ e do fluxo potencial matricial $\left(\phi_{\mathrm{m}}\right)$. A condutividade hidráulica e o fluxo potencial mátrico utilizando-se uma carga hidráulica, podem ser obtidos a partir das Eqs. 15 e 16 (Soil Moisture, 1991).

$$
\begin{gathered}
\mathrm{K}_{0}=\frac{\mathrm{C}_{1} \mathrm{Q}_{1}}{2 \pi \mathrm{H}_{1}^{2}+\pi \mathrm{a}^{2} \mathrm{C}_{1}+2 \pi \frac{\mathrm{H}_{1}}{\alpha_{\mathrm{G}}}} \\
\phi_{\mathrm{m}}=\frac{\mathrm{C}_{1} \mathrm{Q}_{1}}{\left(2 \pi \mathrm{H}_{1}^{2}+\pi \mathrm{a}^{2} \mathrm{C}_{1}\right) \alpha_{\mathrm{G}}+2 \pi \mathrm{H}_{1}}
\end{gathered}
$$

em que:

$\alpha_{\mathrm{G}}$ - pode ser obtido a partir da Tabela 1

Os testes de condutividade hidráulica nas Bacias Experimentais do Jatobá e do Mimoso foram realizados em encostas (em diferentes tipos de solo), em diferentes horizontes, após abertura de trincheiras e caracterização do perfil. Buscou-se encamisar os furos de sondagem com tela de nylon, para evitar desmoronamentos.

Os testes foram realizados em manchas de Argissolos (solos de referência dominantes) que, segundo Santos et al. (2009b), foram classificados como Argissolo Amarelo Eutrófico abruptico (Bacia do Jatobá) e como Argissolo Amarelo Eutrófico típico (Bacia do Mimoso). Buscou-se realizar, no mínimo, três testes de condutividade hidráulica nas camadas de cada área representativa, conduzidos em cada horizonte. Em situações de elevada dispersão de estimativas testes adicionais foram efetuados com o objetivo de reduzir a incerteza nas medições.

O comportamento hidráulico dos solos também foi avaliado em função do tipo de tratamento conservacionista e cobertura vegetal, em parcelas de monitoramento hidrossedimentológico, com diferentes tratamentos e cobertura. Os tratamentos considerados foram: cobertura natural $(\mathrm{CN})$, solo descoberto (SD), cultivo morro abaixo (CMA), palma em curva de nível (PCN) e barramento de pedra com cobertura morta (BPCM), conforme descrito por Santos et al. (2009c).

Realizou-se a análise estatística através do "software" SAS - Statistical Analitical System (SAS, 1998). Para comparação entre as médias das condutividades hidráulicas obtidas nos diferentes tratamentos conservacionistas, adotou-se o teste de Tukey, a um nível de significância de 5\%.
Realizaram-se, no total, 29 testes de condutividade hidráulica no Argissolo Amarelo Eutrófico abruptico (Bacia do Jatobá) em diferentes profundidades (treze a $15 \mathrm{~cm}$; treze a $30 \mathrm{~cm}$ e três na profundidade de $55 \mathrm{~cm}$ ) enquanto no Argissolo Amarelo Eutrófico típico (Bacia do Mimoso) foram realizados 21 testes (sete a $15 \mathrm{~cm}$; dez a $40 \mathrm{~cm}$ e quatro na profundidade de $100 \mathrm{~cm}$ ). Com relação aos testes realizados nas diferentes coberturas vegetais, realizaram-se ainda quinze testes em cada bacia, isto é, três testes em cada tratamento conservacionista.

\section{Resultados E Discussão}

O Argissolo Amarelo Eutrófico Abrúptico (Bacia do Jatobá) apresentou textura Franco Argilosa, nas profundidades superficiais e subsuperficiais, e textura Argilosa para profundidades superiores a $55 \mathrm{~cm}$, exibindo camada de impedimento a $75 \mathrm{~cm}$; já o Argissolo Amarelo Eutrófico típico (Bacia do Mimoso) apresentou textura Franco Argilosa, para as profundidades até $95 \mathrm{~cm}$ e textura Argilosa nas profundidades superiores. A fração argila é dominante nas camadas de profundidade igual ou superior a $30 \mathrm{~cm}$, nas duas Bacias.

As Tabelas de 2 a 5 apresentam a estatística descritiva da textura e do teor de matéria orgânica em diferentes profundidades de perfis característicos em condições naturais e a $15 \mathrm{~cm}$ em tratamentos conservacionistas com diferentes coberturas vegetais.

\section{Bacia Experimental do Jatobá (Argissolo Amarelo Eutrófico Abruptico)}

Observa-se, na Tabela 2, que o teor de areia vai diminuindo com a profundidade, sendo esta uma das características dos Argissolos, que apresentam diferença acentuada de textura entre o horizonte superficial (A) e o horizonte subjacente (B textural) (IPA, 2008). Tal comportamento também foi observado por Santana et al. (2006), em Argissolos do Estado da Bahia e por Perusi \& Carvalho (2007), em Argissolos em Anhumas, SP. O teor de matéria orgânica no perfil do solo também apresentou decaimento com a profundidade, que pode ser explicado com base no equilíbrio que existe entre a deposição de materiais orgânicos (baixa disponibilidade), o efeito da atividade microbiana nas camadas superficiais, a umidade e temperatura do solo tal como, ainda, pelo aumento da densidade, que impede o carreamento da matéria orgânica para as camadas subjacentes. O mesmo comportamento também foi observado por Trindade et al. (2009). 
Tabela 2. Estatística descritiva da textura e matéria orgânica nas diferentes profundidades do Argissolo A marelo Eutrófico Abrúptico (Bacia Experimental do Jatobá)

\begin{tabular}{|c|c|c|c|c|c|c|c|c|c|}
\hline & \multicolumn{3}{|c|}{ Profundidade $20 \mathrm{~cm}$} & \multicolumn{3}{|c|}{ Profundidade $35 \mathrm{~cm}$} & \multicolumn{3}{|c|}{ Profundidade $55 \mathrm{~cm}$} \\
\hline & Areia & Argila & M.0. & Areia & Argila & M.0. & Areia & Argila & M.0. \\
\hline & \multicolumn{9}{|c|}{$\left(\mathrm{g} \mathrm{kg}^{-1}\right)$} \\
\hline MD & 356,53 & 348,61 & 14,12 & 320,63 & 378,36 & 10,54 & 286,87 & 407,59 & 6,31 \\
\hline MED & 342,42 & 357,59 & 13,05 & 342,42 & 374,25 & 10,44 & 275,76 & 407,59 & 6,09 \\
\hline DESVPAD & 66,61 & 30,13 & 3,86 & 53,31 & 35,44 & 3,13 & 19,24 & 16,67 & 1,21 \\
\hline C.V & 0,19 & 0,09 & 0,27 & 0,17 & 0,09 & 0,30 & 0,07 & 0,04 & 0,19 \\
\hline AS & 1,89 & $-1,03$ & 0,79 & $-0,79$ & 0,36 & 0,37 & 1,73 & 0,00 & 0,78 \\
\hline
\end{tabular}

MD - média aritmética; MED - mediana; DESVPAD - desvio-padrão; C.V - coeficiente de variação; AS - assimetria

Tabela 3. Estatística descritiva da textura e do teor de matéria orgânica nos diferentes tratamentos conservacionistas e tipos de cobertura vegetal, na profundidade de $15 \mathrm{~cm}$ (Bacia Experimental do Jatobá)

\begin{tabular}{|c|c|c|c|c|c|c|}
\hline TRAT & $\begin{array}{l}\text { ATRIB } \\
\left(\mathrm{g} \mathrm{kg}^{-1}\right)\end{array}$ & MD & MED & DESVPAD & C.V & AS \\
\hline BPCM & $\begin{array}{l}\text { Areia } \\
\text { Argila } \\
\text { M.0. }\end{array}$ & $\begin{array}{r}265,76 \\
394,25 \\
14,57\end{array}$ & $\begin{array}{r}225,76 \\
407,59 \\
14,14\end{array}$ & $\begin{array}{r}99,02 \\
51,91 \\
1,89\end{array}$ & $\begin{array}{l}0,37 \\
0,13 \\
0,13\end{array}$ & $\begin{array}{r}0,85 \\
-0,56 \\
-0,33\end{array}$ \\
\hline CMA & $\begin{array}{l}\text { Areia } \\
\text { Argila } \\
\text { M.0. }\end{array}$ & $\begin{array}{r}320,20 \\
357,59 \\
16,39\end{array}$ & $\begin{array}{r}325,76 \\
357,59 \\
14,79\end{array}$ & $\begin{array}{r}9,62 \\
16,67 \\
2,95\end{array}$ & $\begin{array}{l}0,03 \\
0,05 \\
0,18\end{array}$ & $\begin{array}{r}-1,73 \\
0,00 \\
1,72\end{array}$ \\
\hline PCN & $\begin{array}{l}\text { Areia } \\
\text { Argila } \\
\text { M.0. }\end{array}$ & $\begin{array}{r}305,76 \\
378,42 \\
14,95\end{array}$ & $\begin{array}{r}319,09 \\
374,25 \\
15,12\end{array}$ & $\begin{array}{r}43,97 \\
25,00 \\
1,88\end{array}$ & $\begin{array}{l}0,14 \\
0,07 \\
0,13\end{array}$ & $\begin{array}{r}-1,54 \\
0,37 \\
-0,23\end{array}$ \\
\hline $\mathrm{CN}$ & $\begin{array}{l}\text { Areia } \\
\text { Argila } \\
\text { M.0. }\end{array}$ & $\begin{array}{r}292,43 \\
365,92 \\
15,44\end{array}$ & $\begin{array}{r}292,43 \\
365,92 \\
15,44\end{array}$ & $\begin{array}{r}116,66 \\
58,33 \\
3,26\end{array}$ & $\begin{array}{l}0,40 \\
0,16 \\
0,21\end{array}$ & $\begin{array}{l}0,00 \\
0,00 \\
0,00\end{array}$ \\
\hline SD & $\begin{array}{l}\text { Areia } \\
\text { Argila } \\
\text { M.0. }\end{array}$ & $\begin{array}{r}403,53 \\
332,59 \\
13,85\end{array}$ & $\begin{array}{r}375,76 \\
340,92 \\
12,72\end{array}$ & $\begin{array}{r}71,23 \\
31,18 \\
2,39\end{array}$ & $\begin{array}{l}0,18 \\
0,09 \\
0,17\end{array}$ & $\begin{array}{r}1,98 \\
-1,65 \\
0,82\end{array}$ \\
\hline
\end{tabular}

TRAT - tratamento; ATRIB - atributo do solo; MD - média aritmética; MED - mediana; DESVPAD - desvio-padrão; C.V - coeficiente de variação; AS - assimetria; BPCM - barramento com pedra e cobertura morta; CMA - cultivo morro abaixo; PCN - palma em curva de nível; CN - cobertura natural; SD - solo descoberto

Tabela 4. Estatística descritiva da textura e do teor de matéria orgânica nas diferentes profundidades do Argissolo Amarelo Eutrófico típico (Bacia Experimental do Mimoso)

\begin{tabular}{|c|c|c|c|c|c|c|c|c|c|}
\hline & \multicolumn{3}{|c|}{ Profundidade $15 \mathrm{~cm}$} & \multicolumn{3}{|c|}{ Profundidade $40 \mathrm{~cm}$} & \multicolumn{3}{|c|}{ Profundidade $100 \mathrm{~cm}$} \\
\hline & Areia & Argila & M.0. & Areia & Argila & M.0. & Areia & Argila & M.0. \\
\hline & & & & & $\left(\mathrm{g} \mathrm{kg}^{-1}\right)$ & & & & \\
\hline MD & 299,57 & 359,97 & 12,63 & 252,43 & 414,25 & 9,51 & 185,76 & 467,59 & 3,67 \\
\hline MED & 309,09 & 374,25 & 11,53 & 242,43 & 415,92 & 9,46 & 179,08 & 474,25 & 3,43 \\
\hline DESVPAD & 40,66 & 35,26 & 2,41 & 43,88 & 22,50 & 4,47 & 15,87 & 29,05 & 1,48 \\
\hline C.V & 0,14 & 0,10 & 0,19 & 0,17 & 0,05 & 0,47 & 0,09 & 0,06 & 0,40 \\
\hline AS & $-0,31$ & $-1,44$ & 0,46 & 1,00 & 0,09 & 1,55 & 1,78 & $-0,68$ & 0,44 \\
\hline
\end{tabular}

O decaimento da matéria orgânica também é influenciado pelo desenvolvimento limitado do sistema radicular vertical da vegetação presente e pelo processo de translocação ao longo do perfil, resultante da percolação.

Dentre os atributos do solo analisados a matéria orgânica foi a que apresentou o maior coeficiente de variação nas três profundidades, com variabilidade média, segundo critérios de Warrick \& Nielsen (1980), enquanto a fração argila apresentou baixa variabilidade. Vieira (1997), estudando a variabilidade espacial das características físicas em Latossolo Roxo, também encontrou coeficientes de variação baixos para teor de argila. A fração areia apresentou média variabilidade nas camadas subsuperficiais (20 e $35 \mathrm{~cm}$ ) e baixa na profundidade de $55 \mathrm{~cm}$. Os valores da mediana e média, para todas as variáveis, estão próximos entre si, indicando distribuições simétricas.

Pode-se observar que o teor de areia apresentou alta variabilidade entre os diferentes tratamentos conservacionistas, tendo a parcela descoberta apresentado o maior percentual de areia (Tabela 3). Este comportamento pode ser explicado pela exposição do solo ao impacto direto da chuva, que promove o carreamento das partículas finas por processo de erosão laminar (Santos et al., 2008). Com relação à matéria orgânica, esperavase um teor maior nos tratamentos de barramento de pedra com cobertura morta (BPCM) e na parcela com cobertura natural $(\mathrm{CN})$, porém não houve diferença expressiva entre os tratamentos. Este comportamento ocorreu, provavelmente, pela elevada taxa de mineralização da matéria orgânica na região, 
Tabela 5. Estatística descritiva da textura e do teor de matéria orgânica nos diferentes tratamentos conservacionistase tipos de cobertura vegetal, na profundidade de $15 \mathrm{~cm}$

\begin{tabular}{|c|c|c|c|c|c|c|}
\hline TRAT & $\begin{array}{l}\text { ATRIB } \\
\left(\mathrm{g} \mathrm{kg}^{-1}\right)\end{array}$ & MD & MED & DESVPAD & C.V & AS \\
\hline BPCM & $\begin{array}{l}\text { Areia } \\
\text { Argila } \\
\text { M.0. }\end{array}$ & $\begin{array}{r}221,60 \\
411,75 \\
13,21\end{array}$ & $\begin{array}{r}209,10 \\
415,92 \\
13,49\end{array}$ & $\begin{array}{r}49,77 \\
28,46 \\
2,17\end{array}$ & $\begin{array}{l}0,22 \\
0,07 \\
0,16\end{array}$ & $\begin{array}{r}1,38 \\
-0,75 \\
-0,37\end{array}$ \\
\hline CMA & $\begin{array}{l}\text { Areia } \\
\text { Argila } \\
\text { M.0. }\end{array}$ & $\begin{array}{r}279,93 \\
370,09 \\
15,23\end{array}$ & $\begin{array}{r}267,43 \\
374,25 \\
15,01\end{array}$ & $\begin{array}{r}43,83 \\
34,36 \\
1,45\end{array}$ & $\begin{array}{l}0,16 \\
0,09 \\
0,10\end{array}$ & $\begin{array}{r}1,44 \\
-0,71 \\
0,84\end{array}$ \\
\hline PCN & $\begin{array}{l}\text { Areia } \\
\text { Argila } \\
\text { M.0. }\end{array}$ & $\begin{array}{r}297,98 \\
352,03 \\
16,97\end{array}$ & $\begin{array}{r}325,76 \\
340,92 \\
16,53\end{array}$ & $\begin{array}{r}63,10 \\
34,69 \\
2,42\end{array}$ & $\begin{array}{l}0,21 \\
0,10 \\
0,14\end{array}$ & $\begin{array}{r}-1,60 \\
1,29 \\
0,78\end{array}$ \\
\hline $\mathrm{CN}$ & $\begin{array}{l}\text { Areia } \\
\text { Argila } \\
\text { M.O. }\end{array}$ & $\begin{array}{r}275,76 \\
363,14 \\
12,69\end{array}$ & $\begin{array}{r}275,76 \\
374,25 \\
12,62\end{array}$ & $\begin{array}{r}33,33 \\
34,69 \\
0,13\end{array}$ & $\begin{array}{l}0,12 \\
0,10 \\
0,01\end{array}$ & $\begin{array}{r}0,00 \\
-1,29 \\
1,73\end{array}$ \\
\hline SD & $\begin{array}{l}\text { Areia } \\
\text { Argila } \\
\text { M.O. }\end{array}$ & $\begin{array}{r}285,28 \\
374,25 \\
12,14\end{array}$ & $\begin{array}{r}275,76 \\
374,25 \\
11,96\end{array}$ & $\begin{array}{r}47,00 \\
19,24 \\
3,22\end{array}$ & $\begin{array}{l}0,16 \\
0,05 \\
0,26\end{array}$ & $\begin{array}{r}0,18 \\
-0,91 \\
-0,03\end{array}$ \\
\hline
\end{tabular}

TRAT - tratamento; ATRIB - atributo do solo; MD - média aritmética; MED - mediana; DESVPAD - desvio- padrão; C.V - coeficiente de variação; AS- assimetria; MIN- mínimo; MAX- máximo; CONTcontagem (número de amostras); BPCM - barramento com pedra e cobertura morta; CMA - cultivo morro abaixo; PCN - palma em curva de nível; CN - cobertura natural; SD - solo descoberto

precipitação, umidade e temperatura do solo uma vez que o solo foi coletado três meses após término do período chuvoso (outubro de 2009). Outro fator que deve ser levado em consideração é o tempo de intervenção na área (uso de práticas conservacionistas, apenas seis meses). Antes das intervenções a área se encontrava na mesma condição (desmatada).

\section{Bacia Experimental do Mimoso (Argissolo Amarelo Eutrófico típico)}

Observa-se, na Tabela 4, que o comportamento da textura e da matéria orgânica do Argissolo Amarelo Eutrófico típico, apresentou similaridade ao comportamento do Argissolo Amarelo Eutrófico Abruptico, com redução da quantidade da areia e de matéria orgânica com a profundidade. Os valores da mediana e média para as variáveis, também ficaram próximos, indicando distribuições simétricas. Adotando-se o critério de classificação para o coeficiente de variação $(\mathrm{CV})$, proposto por Warrick \& Nielsen (1980) para a medida de dispersão, verificouse que a matéria orgânica apresentou variabilidade média nas três profundidades investigadas.

Observa-se ainda, na Tabela 4, que o teor de argila apresentou baixa variabilidade mas, para o teor de areia, apresentou variabilidade média nas camadas mais sub-superficiais (15 e 40 $\mathrm{cm}$ ) e baixa na profundidade de $100 \mathrm{~cm}$. A baixa variabilidade na profundidade de $100 \mathrm{~cm}$ está ligada, possivelmente, à condição de preservação da estrutura do solo. Coelho Filho et al. (2001) também constataram, em estudo da variabilidade espacial das variáveis físico-hídricas de um solo classificado como Terra Roxa Estruturada, baixa variabilidade para a argila e variabilidade média para a areia, enquanto Lima et al. (2006) encontraram, em um Cambissolo, variabilidade média para a argila e baixa para areia total.

Tem-se, na Tabela 5, que o teor de areia também apresentou alta variabilidade entre os diferentes tratamentos conservacionistas; a parcela com palma cultivada em nível foi a que apresentou o maior percentual de areia; com relação à matéria orgânica se esperava, novamente, maior teor nos tratamentos de barramento de pedra com cobertura morta (BPCM) e na parcela com cobertura natural $(\mathrm{CN})$, porém não houve diferença expressiva entre os tratamentos, o que foi explicado pela mesma condição da Bacia do Jatobá.

\section{Caracterização da condutividade hidráulica em diferentes profundidades}

As análises a seguir apresentam o comportamento estatístico da condutividade hidráulica do solo saturado nas Bacias Experimentais do Jatobá e do Mimoso, ao longo do perfil. Observa-se elevada variabilidade da condutividade hidráulica nas duas bacias, com exceção das profundidades com estrutura mais preservada. Verificou-se, nas bacias em estudo, que os valores de $\mathrm{K}_{0}$ apresentaram similaridade nas camadas subsuperficiais (30 a $40 \mathrm{~cm}$ ), possibilitando a consideração de um valor médio de $7,84 \mathrm{~cm} \mathrm{~h}^{-1}$, para fins de modelagem hidrológica.

\section{Estatística descritiva da condutividade hidráulica do solo saturado das Bacias Experimentais do Jatobá e do Mimoso}

A Tabela 6 descreve o comportamento estatístico da condutividade hidráulica do solo saturado em diferentes profundidades, nas Bacias Experimentais do Jatobá e do Mimoso, Pesqueira, PE. As camadas até $50 \mathrm{~cm}$ apresentaram alta variabilidade; assim, para se obter resultados mais representativos foi necessário realizar um número maior de testes.

Nota-se ainda, na Tabela 5, que a condutividade hidráulica do solo saturado $\left(\mathrm{K}_{0}\right)$ média na bacia do Jatobá foi decrescendo ao longo do perfil. Verificou-se, em cálculos preliminares e observações de campo, que as profundidades de 15 e $35 \mathrm{~cm}$ apresentaram alta variabilidade; a condutividade hidráulica do solo saturado mínima foi semelhante nas três profundidades porém os valores máximos encontrados nessas profundidades foram 10,49 e 11,17 vezes superiores aos valores mínimos, respectivamente, comportamento que pode ser explicado pela heterogeneidade e uso do solo, visto que a área já foi desmatada 
Tabela 6. Estatística descritiva da condutividade hidráulica do solo saturado $\left(\mathrm{cm} \mathrm{h}^{-1}\right)$ em diferentes profundidades

\begin{tabular}{|c|c|c|c|c|c|c|}
\hline \multirow{3}{*}{$\begin{array}{l}\text { Estatística } \\
\text { descritiva }\end{array}$} & \multicolumn{3}{|c|}{$\begin{array}{l}\text { Bacia do J atobá } \\
\text { Argissolo Amarelo } \\
\text { Eutrófico abrúptico }\end{array}$} & \multicolumn{3}{|c|}{$\begin{array}{l}\text { Bacia do Mimoso } \\
\text { Argissolo Amarelo } \\
\text { Eutrófico típico }\end{array}$} \\
\hline & \multicolumn{6}{|c|}{ Profundidade $(\mathrm{cm})$} \\
\hline & 15 & 35 & 55 & 15 & 40 & 100 \\
\hline MG & 8,43 & 7,97 & 1,44 & 13,39 & 7,32 & 8,15 \\
\hline MED & 8,73 & 8,30 & 1,49 & 12,51 & 7,38 & 8,06 \\
\hline DESVPAD & 4,13 & 4,55 & 0,21 & 6,96 & 4,21 & 0,66 \\
\hline C.V. & 0,49 & 0,57 & 0,15 & 0,52 & 0,57 & 0,08 \\
\hline AS & 0,12 & $-0,12$ & $-1,00$ & 0,99 & 0,11 & 0,32 \\
\hline MIN & 1,49 & 1,28 & 1,20 & 6,78 & 1,67 & 7,57 \\
\hline MAX & 15,63 & 14,30 & 1,62 & 25,74 & 13,87 & 8,91 \\
\hline CONT & 13,00 & 13,00 & 3,00 & 7,00 & 10,00 & 4,00 \\
\hline N.C $(95 \%)$ & 2,49 & 2,75 & 0,53 & 6,43 & 3,01 & 1,05 \\
\hline
\end{tabular}

MG - média geométrica; MED - mediana; DESVPAD - desvio-padrão; C.V - coeficiente de variação; AS - assimetria; MIN - mínimo; MAX - máximo; CONT - contagem (número de amostras); N.C nível de confiança a $95 \%$

e mobilizada através de tração animal fato que, possivelmente, promoveu mudanças na estrutura do solo, na geometria e continuidade dos poros, influenciando diretamente a $\mathrm{K}_{0}$.

Com relação à Bacia do Mimoso, a condutividade hidráulica média do solo saturado foi maior na camada superficial de 15 $\mathrm{cm}$, diminuindo na profundidade de $40 \mathrm{~cm}$ e voltando a aumentar na profundidade de $100 \mathrm{~cm}$; as camadas mais superficiais (15 e $40 \mathrm{~cm}$ ) apresentaram alta variabilidade, o que pode ser um indicativo do manejo e uso do solo, uma vez que a mesma já foi mecanizada para preparo agrícola; a condutividade hidráulica do solo saturado na profundidade de $100 \mathrm{~cm}$ apresentou baixo desvio padrão e os valores máximos e mínimos ficaram próximos da média, comportamento que pode estar associado à estrutura do solo que, a esta profundidade, se encontra preservada.

\section{Caracterização da condutividade hidráulica em diferentes profundidades}

A condutividade hidráulica saturada do Argissolo Amarelo Eutrófico Abrúptico diminui com a profundidade (Tabela 6). Bastos (2004), avaliando a condutividade hidráulica com permeâmetro de Guelph em diferentes profundidades de um Neossolo Flúvico Tb Eutrófico, presente na Bacia Experimental do Mimoso, encontrou o mesmo comportamento, com exceção da profundidade de $30 \mathrm{~cm}$ que indicou condutividade hidráulica do solo saturado menor que as demais. Segundo o autor, o resultado está relacionado à compactação e ao acúmulo de matéria orgânica; tal comportamento se mostrou diferente dos encontrados por diversos autores, dentre eles Carvalho et al. (2007), em Latossolo Vermelho-Amarelo, Aragão Junior et al. (2007) em Argissolo Vermelho-Amarelo.

A condutividade hidráulica do Argissolo Amarelo Eutrófico Típico também foi superior na primeira camada, diminuiu na profundidade de $40 \mathrm{~cm}$ e voltou a aumentar a $100 \mathrm{~cm}$, comportamento que diferiu do encontrado na Bacia do Jatobá, que apresentou diminuição da condutividade com a profundidade (Tabela 6). Mesquita \& Moraes (2004) comentam que o efeito da estrutura e em especial dos poros de grandes dimensões, permite que um solo argiloso tenha valores de condutividades hidráulicas saturadas similares aos dos solos arenosos.

$\mathrm{Na}$ Tabela 7 verifica-se a estatística descritiva da condutividade hidráulica do solo saturado $\left(\mathrm{K}_{0}\right)$, do potencial de fluxo mátrico $\left(\phi_{\mathrm{m}}\right)$ e do parâmetro alfa $\left(\alpha_{\mathrm{G}}\right)$ da Bacia do Jatobá e que o valor de alfa tendeu a diminuir com a profundidade; tal comportamento corrobora com o resultado encontrado por Ragab \& Cooper (1993), ao observarem que os valores de alfa $\left(\alpha_{G}\right)$ diminuíram com a profundidade nas áreas de pastagem e terras aradas e tenderam a ficar constantes, em condição de floresta.

Observa-se, na Tabela 8, o comportamento estatístico da condutividade hidráulica do solo saturado $\left(\mathrm{K}_{0}\right)$, do potencial de fluxo mátrico $\left(\phi_{\mathrm{m}}\right)$ e do parâmetro alfa $\left(\alpha_{\mathrm{G}}\right)$ da Bacia do Mimoso enquanto o parâmetro alfa aumentou com a profundidade sinalizando o maior coeficiente de variação na profundidade de $40 \mathrm{~cm}$; mesmo assim, o maior desvio do alfa em relação à média aritmética ocorreu na profundidade de 100 $\mathrm{cm}$. Os valores médios de condutividade hidráulica do solo saturado nas profundidades de 40 e $100 \mathrm{~cm}$ ocorreram em escalas semelhantes.

A partir das estimativas apresentadas nas Tabelas 7 e 8 foi possível gerar as curvas de condutividade hidráulica do solo não saturado (Figura 2A), nas diferentes profundidades, através da Eq. 2, proposta por Gardner (1958).

Pode-se observar, na Figura 2A, que o comportamento da condutividade hidráulica do solo não saturado em função da tensão $(K(\psi))$ nas profundidades de 35 e $55 \mathrm{~cm}$ foi semelhante, com as maiores condutividades hidráulicas ocorrendo a $35 \mathrm{~cm}$ de profundidade; já na profundidade de $15 \mathrm{~cm}$ nota-se uma

Tabela 7. Estatística descritiva da condutividade hidráulica do solo saturado $\left(\mathrm{K}_{0}\right)$, potencial de fluxo mátrico $\left(\phi_{\mathrm{m}}\right)$ e parâmetro alfa $\left(\alpha_{G}\right)$ da função de Gardner (1958) da Bacia do Jatobá

\begin{tabular}{|c|c|c|c|c|c|c|c|c|c|}
\hline & \multicolumn{3}{|c|}{$15 \mathrm{~cm}$} & \multicolumn{3}{|c|}{$35 \mathrm{~cm}$} & \multicolumn{3}{|c|}{$55 \mathrm{~cm}$} \\
\hline & $\begin{array}{c}\mathrm{K}_{0} \\
\left(\mathrm{~cm} \mathrm{~s}^{-1}\right)\end{array}$ & $\begin{array}{c}\phi_{\mathrm{m}} \\
\left(\mathrm{cm}^{2} \mathrm{~s}^{-1}\right)\end{array}$ & $\begin{array}{c}\alpha_{G} \\
\left(\mathrm{~cm}^{-1}\right)\end{array}$ & $\begin{array}{c}\mathrm{K}_{0} \\
\left(\mathrm{~cm} \mathrm{~s}^{-1}\right)\end{array}$ & $\begin{array}{c}\phi_{\mathrm{m}} \\
\left(\mathrm{cm}^{2} \mathbf{s}^{-1}\right)\end{array}$ & $\begin{array}{c}\alpha_{G} \\
\left(\mathrm{~cm}^{-1}\right)\end{array}$ & $\begin{array}{c}\mathrm{K}_{0} \\
\left(\mathrm{~cm} \mathrm{~s}^{-1}\right)\end{array}$ & $\begin{array}{c}\phi_{\mathrm{m}} \\
\left(\mathrm{cm}^{2} \mathbf{s}^{-1}\right)\end{array}$ & $\begin{array}{c}\alpha_{G} \\
\left(\mathrm{~cm}^{-1}\right)\end{array}$ \\
\hline MD & 0,00234 & 0,01096 & 0,62100 & 0,00221 & 0,01207 & 0,45209 & 0,00040 & 0,00362 & 0,34214 \\
\hline MG & 0,00201 & 0,00454 & 0,44277 & 0,00177 & 0,00651 & 0,27236 & 0,00040 & 0,00178 & 0,22293 \\
\hline DESVPAD & 0,00115 & 0,01934 & 0,48711 & 0,00126 & 0,01521 & 0,45918 & 0,00006 & 0,00491 & 0,27405 \\
\hline CV & 0,48962 & 1,76391 & 0,78439 & 0,57037 & 1,26082 & 1,01568 & 0,14839 & 1,35824 & 0,80099 \\
\hline AS & 0,12384 & 2,88568 & 1,76410 & $-0,12092$ & 2,51362 & 1,23365 & $-1,00183$ & 1,72986 & $-0,71623$ \\
\hline MIN & 0,00041 & 0,00060 & 0,03435 & 0,00036 & 0,00091 & 0,05929 & 0,00033 & 0,00070 & 0,04844 \\
\hline MAX & 0,00434 & 0,07056 & 1,95740 & 0,00397 & 0,05770 & 1,44784 & 0,00045 & 0,00929 & 0,59103 \\
\hline CONT & 13 & 13 & 13 & 13 & 13 & 13 & 3 & 3 & 3 \\
\hline
\end{tabular}

MD - média aritmética; MG - média geométrica; DESVPAD - desvio-padrão; C.V - coeficiente de variação; AS - assimetria; MIN - mínimo; MAX - máximo; CONT - contagem (número de amostras); $\alpha_{G}$ - parâmetro alfa da equação de Gardner (1958); $\phi_{\mathrm{m}}$ - fluxo potencial mátrico; $\mathrm{K}_{0}$ - condutividade hidráulica do solo saturado 
Tabela 8. Estatística descritiva da condutividade hidráulica do solo saturado $\left(\mathrm{K}_{0}\right)$, potencial de fluxo mátrico $\left(\phi_{\mathrm{m}}\right)$ e parâmetro alfa $\left(\alpha_{G}\right)$ da função de $G$ ardner (1958) da Bacia do M imoso

\begin{tabular}{|c|c|c|c|c|c|c|c|c|c|}
\hline & \multicolumn{3}{|c|}{$15 \mathrm{~cm}$} & \multicolumn{3}{|c|}{$40 \mathrm{~cm}$} & \multicolumn{3}{|c|}{$100 \mathrm{~cm}$} \\
\hline & $\begin{array}{c}\mathrm{K}_{0} \\
\left(\mathrm{~cm} \mathrm{~s}^{-1}\right)\end{array}$ & $\begin{array}{c}\phi_{\mathrm{m}} \\
\left(\mathrm{cm}^{2} \mathbf{s}^{-1}\right)\end{array}$ & $\begin{array}{c}\alpha_{G} \\
\left(\mathrm{~cm}^{-1}\right)\end{array}$ & $\begin{array}{c}\mathrm{K}_{0} \\
\left(\mathrm{~cm} \mathrm{~s}^{-1}\right)\end{array}$ & $\begin{array}{c}\phi_{\mathrm{m}} \\
\left(\mathrm{cm}^{2} \mathrm{~s}^{-1}\right)\end{array}$ & $\begin{array}{c}\alpha_{G} \\
\left(\mathrm{~cm}^{-1}\right)\end{array}$ & $\begin{array}{c}\mathrm{K}_{0} \\
\left(\mathrm{~cm} \mathrm{~s}^{-1}\right)\end{array}$ & $\begin{array}{c}\phi_{\mathrm{m}} \\
\left(\mathrm{cm}^{2} \mathrm{~s}^{-1}\right)\end{array}$ & $\begin{array}{c}\alpha_{G} \\
\left(\mathrm{~cm}^{-1}\right)\end{array}$ \\
\hline MD & 0,00372 & 0,00854 & 0,47367 & 0,00203 & 0,01000 & 0,88810 & 0,00226 & 0,00114 & 4,97108 \\
\hline MG & 0,00333 & 0,00758 & 0,43965 & 0,00168 & 0,00472 & 0,35578 & 0,00226 & 0,00073 & 3,11007 \\
\hline DESVPAD & 0,00193 & 0,00437 & 0,18433 & 0,00117 & 0,01168 & 1,57617 & 0,00018 & 0,00111 & 4,42333 \\
\hline C.V & 0,51965 & 0,51121 & 0,38914 & 0,57445 & 1,16765 & 1,77477 & 0,08091 & 0,96963 & 0,88981 \\
\hline AS & 0,99012 & 0,59509 & $-0,10030$ & 0,11311 & 1,59646 & 2,78824 & 0,31976 & 0,86061 & $-0,01856$ \\
\hline MIN & 0,00188 & 0,00310 & 0,23135 & 0,00046 & 0,00049 & 0,10234 & 0,00210 & 0,00027 & 0,82021 \\
\hline MAX & 0,00715 & 0,01446 & 0,70059 & 0,00385 & 0,03679 & 5,21472 & 0,00247 & 0,00258 & 8,84051 \\
\hline CONT & 7 & 7 & 7 & 10 & 10 & 10 & 4 & 4 & 4 \\
\hline
\end{tabular}

MD - média aritmética; MG - média geométrica; DESVPAD - desvio-padrão; C.V - coeficiente de variação; AS - assimetria; MIN- mínimo; MAX - máximo; CONT - contagem (número de amostras); $\alpha_{G}$ - parâmetro alfa da equação de Gardner (1958); $\phi_{\mathrm{m}}$ - fluxo potencial mátrico; $\mathrm{K}_{0}$ - condutividade hidráulica do solo saturado

A.

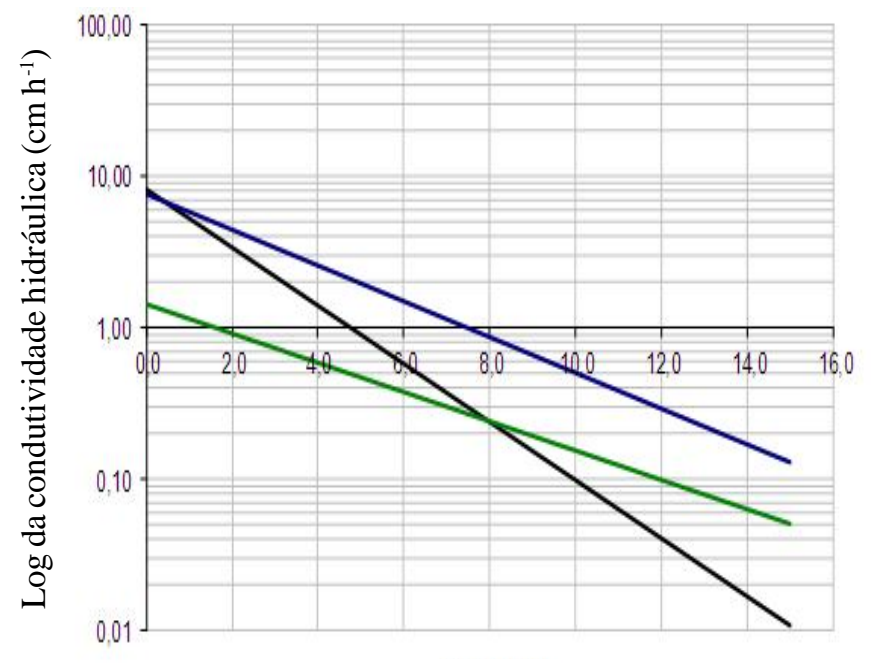

Tensão (cm)

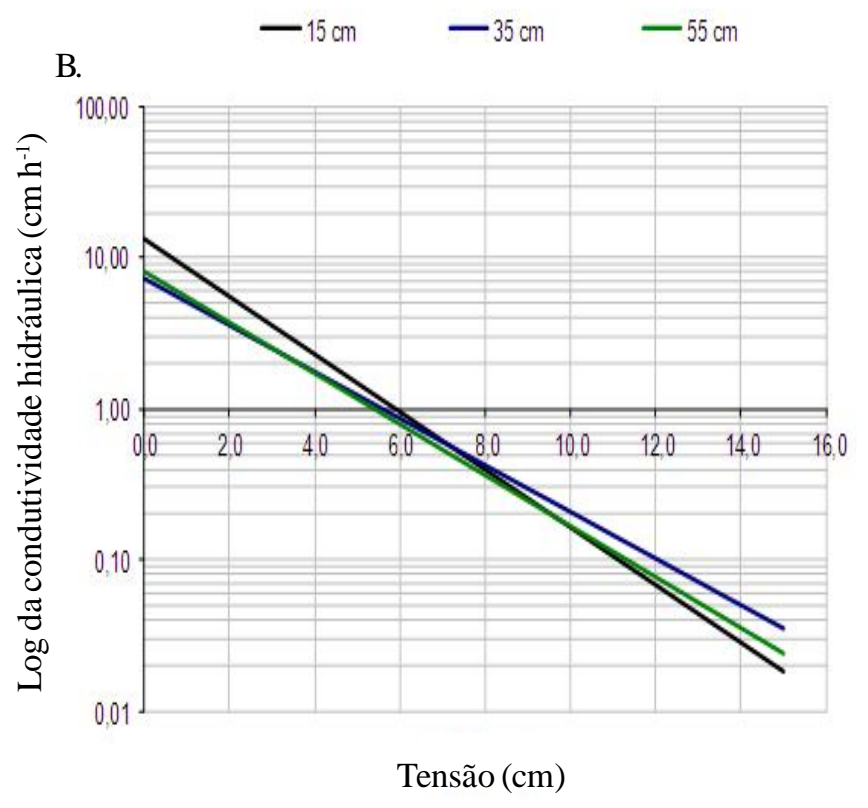

$-15 \mathrm{~cm} \quad-40 \mathrm{~cm} \quad-100 \mathrm{~cm}$

Figura 2. Curvas logaritmadas da condutividade hidráulica do solo não saturado nas diferentes profundidades da Bacia do Jatobá (A) e da Bacia do Mimoso (B), obtidas pela equação de G ardner (1958) queda mais acentuada da condutividade hidráulica do solo não saturado, em relação às outras profundidades.

Na Figura 2B pode-se notar comportamento diferenciado em relação ao da Bacia do Jatobá; por exemplo, para a tensão de $10 \mathrm{~cm}$ tem-se que a condutividade hidráulica não saturada na Bacia do Mimoso é superior na camada superficial ao valor da Bacia do Jatobá. Notam-se valores inferiores na Bacia do Jatobá em relação à Bacia do Mimoso, com exceção da profundidade de $35 \mathrm{~cm}$.

\section{Caracterização da condutividade hidráulica em diferentes tra- tamentos e tipos de cobertura vegetal}

Para realizar o teste de média do logaritmo da condutividade hidráulica do solo saturado entre os tratamentos conservacionistas, foram adotadas três repetições por tratamento, Tabela 9.

Tabela 9. Teste de médias em diferentes tratamentos conservacionistas

\begin{tabular}{|c|c|c|c|c|c|}
\hline \multirow{3}{*}{ Tratamento } & \multirow{3}{*}{$\mathbf{N}$} & \multicolumn{2}{|c|}{ Bacia do J atobá } & \multicolumn{2}{|c|}{ Bacia do Mimoso } \\
\hline & & \multicolumn{4}{|c|}{$\mathrm{K}_{0}\left(\mathrm{~cm} \mathrm{~h}^{-1}\right)$} \\
\hline & & Média & Desvpad & Média & Desvpad \\
\hline BPCM & 3 & $4,35 \mathrm{a}$ & 0,487 & $6,25 a$ & 2,341 \\
\hline CMA & 3 & $11,48 a$ & 5,294 & $9,15 \mathrm{a}$ & 1,167 \\
\hline PCN & 3 & $6,26 a$ & 0,971 & $8,36 \mathrm{a}$ & 2,095 \\
\hline $\mathrm{CN}$ & 3 & $10,40 \mathrm{a}$ & 1,407 & $10,09 a$ & 1,943 \\
\hline PD & 3 & $8,77 \mathrm{a}$ & 0,062 & $7,44 a$ & 0,286 \\
\hline
\end{tabular}

* BPCM - Barramento com pedra e cobertura morta, CMA - Cultivo morro abaixo, PCN - Palma em curva de nível, CN - Cobertura natural e PD - Parcela descoberta. * Médias seguidas de letras minúsculas na mesma coluna e na mesma linha, não diferem entre si a nível de $5 \%$ de significância, pelo teste de Tukey. DESVPAD - desvio padrão

Santos et al. (2008) constataram, em estudo de erosão hídrica em diferentes tipos de cobertura sobre Neossolo Flúvico no semiárido (Bacia do Mimoso), que as taxas de infiltração apresentaram diferença significativa entre as médias dos tratamentos parcela descoberta (PD), cultivo morro abaixo (MA), plantio em curva de nível (PCN), barramento em pedra com cobertura morta (BPCM) e cobertura natural $(\mathrm{CN})$, com valores de 0,$4 ; 1,4 ; 1,8 ; 5,5$ e $5,4 \mathrm{~cm} \cdot \mathrm{h}^{-1}$ para a taxa de infiltração básica. Já o BPCM e a CN apontaram valores significativamente superiores aos demais. Considerando que o valor da $\mathrm{K}_{0}$ é aproximadamente o valor da taxa de infiltração básica, compararam-se os valores encontrados por Santos et al. (2008) 
com os valores de $\mathrm{K}_{0}$ encontrados neste trabalho (Tabela 9). Pode-se observar que os valores de $\mathrm{K}_{0}$ do presente estudo foram superiores aos encontrados por Santos et al. (2008). Deve-se levar em consideração, no entanto, que o método utilizado no presente trabalho foi do permeâmetro de Guelph, enquanto Santos et al. (2008) utilizaram o método do simulador de chuva.

Neste trabalho, diferentemente de Santos et al. (2008), não se verificou diferença significativa para $\mathrm{K}_{0}$ entre os diferentes tratamentos; é provável que as práticas conservacionistas ainda não tivessem promovido mudanças significativas na estrutura do solo, a ponto de alterar sua infiltrabilidade na camada de 15 $\mathrm{cm}$, em função de se tratar do primeiro ano de intervenção nas áreas em estudo. Adicionalmente, deve-se ressaltar que os testes com o permeâmetro de Guelph foram realizados na profundidade de $15 \mathrm{~cm}$ e as avaliações, por sua vez, efetuadas por Santos et al. (2008) e Santos et al. (2009a) que, adotando simulador de chuva, sofreram forte influência da rugosidade das camadas mais superficiais do solo contribuindo, assim, para a diferença de resultados entre as duas metodologias.

\section{Curva de retenção de umidade do solo}

Com base no ajuste da curva de retenção de umidade do solo e segundo o modelo proposto por van Genuchten (1980), observa-se que a umidade de saturação para os solos das Bacias Experimentais do Jatobá e do Mimoso se aproxima de 30\%.

As curvas características indicaram comportamento semelhante, sobretudo nas profundidades de 35 e $55 \mathrm{~cm}$ (Bacia do Jatobá) e na de $40 \mathrm{~cm}$ (Bacia do Mimoso). A profundidade de $15 \mathrm{~cm}$ (Bacia do Mimoso) foi a que apresentou a menor capacidade de retenção de água no solo. De forma geral, verificou-se um ajuste adequado entre os dados experimentais e o modelo de van Genuchten.

A Tabela 10 apresenta os valores dos parâmetros de ajuste $\left(\alpha_{\mathrm{vG}}, \mathrm{n}\right.$ e $\left.\mathrm{m}\right)$ estimados utilizando-se o funcional de van Genuchten (Eq. 1). Em ambas as bacias o parâmetro alfa aumenta com a profundidade.

Tabela 10. Parâmetros de ajuste estimados pela equação de van G enuchten (1980)

\begin{tabular}{|c|c|c|c|c|c|c|}
\hline \multirow{2}{*}{ Bacia } & \multirow{2}{*}{$\begin{array}{l}\text { Prof. } \\
(\mathrm{cm})\end{array}$} & $\theta \mathrm{s}$ & $\theta r$ & \multirow{2}{*}{$\begin{array}{c}\alpha_{\mathrm{vG}} \\
\mathrm{kPa}^{-1}\end{array}$} & \multirow{2}{*}{$\mathbf{N}$} & \multirow{2}{*}{ m } \\
\hline & & \multicolumn{2}{|c|}{$\mathrm{cm}^{3} \mathrm{~cm}^{-3}$} & & & \\
\hline J atobá & $\begin{array}{l}35 \\
55\end{array}$ & $\begin{array}{l}0,300 \\
0,300\end{array}$ & $\begin{array}{l}0,000 \times 10^{+0} \\
2,000 \times 10^{-6}\end{array}$ & $\begin{array}{l}3,620 \\
5,018\end{array}$ & $\begin{array}{l}1,131 \\
1,127\end{array}$ & $\begin{array}{l}0,116 \\
0,113\end{array}$ \\
\hline Mimoso & $\begin{array}{l}15 \\
40\end{array}$ & $\begin{array}{l}0,300 \\
0,300\end{array}$ & $\begin{array}{l}2,368 \times 10^{-2} \\
0,000 \times 10^{+0}\end{array}$ & $\begin{array}{l}2,187 \\
3,631\end{array}$ & $\begin{array}{l}1,176 \\
1,132\end{array}$ & $\begin{array}{l}0,150 \\
0,117\end{array}$ \\
\hline
\end{tabular}

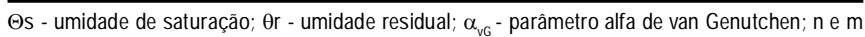
- parâmetros de ajuste experimental

\section{CONClusÕES}

1. Nas Bacias do Jatobá e Mimoso a condutividade hidráulica do solo saturado apresentou alta variabilidade, nas camadas de até $50 \mathrm{~cm}$.

2. Na Bacia do Jatobá ocorreu um decaimento da condutividade hidráulica do solo saturado com a profundidade, o que não foi observado na Bacia do Mimoso.

3. Devido à similaridade dos valores de $\mathrm{K}_{0}$ encontrados nas camadas subsuperficiais ( $30 \mathrm{a} 40 \mathrm{~cm}$ ) em ambas as bacias, pode- se considerar um valor médio de $7,84 \mathrm{~cm} \mathrm{~h}^{-1}$ para fins de modelagem hidrológica.

4. Em ambas as bacias o teor de areia diminui com a profundidade e as curvas de retenção de umidade do solo apresentaram comportamento semelhante para as profundidades investigadas.

5. Não houve diferença significativa da $K_{0}$ nos diferentes tratamentos conservacionistas avaliados.

\section{AgRAdECIMENTOS}

Os autores agradecem o apoio da Finep e CNPq/CTHidro.

\section{LITERATURA CITADA}

Aragão Júnior, T. C. A.; Magalhães, C. A.; Castro, P. T. Determinação da condutividade hidráulica em um solo podzólico-Vermelho-Amarelo em condições de campo. Pesquisa Agropecuária Brasileira, v.18, p.805-810, 2007.

Assis, R. L.; Lanças, K. P. Avaliação dos atributos físicos de um Nitossolo Vermelho Distrófico sob sistema plantio direto, preparo convencional e mata nativa. Revista Brasileira de Ciência do Solo, v.29, p.515-522, 2005.

Bagarello, V.; Di Stefano, C.; Ferro, V.; Iovino, M.; Sgroi, A. Physical and hydraulic characterization of a clay soil at the plot scale. Journal of Hydrology, v.387, p.54-64, 2010.

Bastos, D. C. O. Manejo da salinidade em irrigação localizada: Análise da alternativa de lixiviação incompleta. Recife: UFRPE, 2004. 68p. Dissertação Mestrado

Carvalho, L. A.; Libardi, P. L.; Rocha, G. C.; Cruz, A. C. R. Caracterização hidráulica de um Latossolo vermelho associada à caracterização pedológica do perfil. Ciência Rural, v.37, p.1008-1013, 2007.

Coelho Filho, M. A.; Rubens D. Coelho, R. D.; Gonçalves, A. C. A. Variabilidade espacial de variáveis físico-hídricas do solo em um pomar de lima ácida Tahiti, irrigado por microaspersão. Revista Brasileira de Engenharia Agrícola e Ambiental, v.5, p.239-246, 2001.

Eguchi, E. S.; Silva, E. L.; Oliveira, M. S. Variabilidade espacial da condutividade hidráulica do solo saturado e da taxa de infiltração básica determinadas "in situ”. Ciência e Agrotecnologia, p.1607-1613, 2003.

Elrick, D. E.; Reynolds, W. D.; Tan, K. A. Hydraulic conductivity measurements in the unsaturated zone using improved well analysis. Groundwater Monitoring Review, v.9, p.184-193, 1989.

EMBRAPA - Empresa Brasileira de Pesquisa Agropecuária. Manual de métodos de análise de solo. 2.ed. Rio de Janeiro: Centro Nacional de Pesquisa de Solos, 1997. 212p.

Gardner, W. R. Some steady state solutions of the unsaturated moisture flow equation with application to evaporation from a water table. Soil Science, v.85, p.228-232, 1958.

IPA - Instituto Agronômico de Pernambuco. Recomendações de adubação para o Estado de Pernambuco: $2^{\mathrm{a}}$ aproximação. 3.ed. Revisada, Recife, 2008. 212p. 
Lima, C. L. R.; Reinert, D. J.; Reichert, J. M.; Suzuki, E. A. S.; Gubiani, P. I. Qualidade físico-hídrica e rendimento de soja (Glycine max L.) e feijão (Phaseolus vulgaris L.) de um Argissolo Vermelho Distrófico sob diferentes sistemas de manejo. Ciência Rural, v.36, p.1172-1178, 2006.

Mesquita, M. G. B. F.; Moraes, S. O. A dependência entre a condutividade hidráulica saturada e atributos físicos do solo. Ciência Rural, v.34, p.963-969, 2004.

Montenegro, S. M. G. L.; Montenegro, A. A. A. Aproveitamento sustentável de aquííferos aluviais no semi-árido. In: Cabral, J. J. S.; Ferreira, J. P. C. L.; Montenegro, S. M. G. L.; Costa,W. (ed.). Água subterrânea: aquíf́eros costeiros e aluviões, vulnerabilidade e aproveitamento. Recife: UFPE, 2004. cap.2, p.61-117.

Perusi, M. C.; Carvalho, W. A. Avaliação da estabilidade de agregados de argissolos em diferentes sistemas de uso e manejo no município de Anhumas-SP. Energia na Agricultura, v.22, p.94-111, 2007.

Ragab, R.; Cooper, J. D. Variability of unsaturated zone water transport parameters: implications for hydrological modelling. 1. In situ measurements. Journal of Hydrology, v.148, p.109-131, 1993.

Reynolds, W. D.; Elrick, D. E. In situ measurement of fieldsaturated hydraulic conductivity, sorptivity, and the $\alpha$ parameter using the Guelph permeameter. Soil Science, v.140, p.292-302, 1985.

Reynolds, W. D.; Elrick, D. E. A method for simultaneous insitu measurement in the vadose zone of field-saturated hydraulic conductivity, sorptivity and the conductivitypressure head relationship. Ground Water Monitoring Review, v.6, p.84-95, 1986.

Santana, M. B; Souza, L. S.; Souza, L. D.; Fontes, L. E. F. Atributos físicos do solo e distribuição do sistema radicular de citros como indicadores de horizontes coesos em dois solos de tabuleiros costeiros do Estado da Bahia. Revista Brasileira de Ciência do Solo, v.30, p.1-12, 2006.

Santos, T. E. M.; Montenegro, A. A. A.; Silva Junior, V. P. Erosão hídrica e perda de carbono orgânico em diferentes tipos de cobertura do solo no semiárido, em condições de chuva simulada. Revista Brasileira de Recursos Hídricos, v.13, p.113-125, 2008.

Santos, T. E. M.; Montenegro, A. A. A.; Pedrosa, E. M. R. Características hidráulicas e perdas de solo e água sob cultivo do feijoeiro no semiárido. Revista Brasileira de Engenharia Agrícola e Ambiental, v.13, p.217-225, 2009a.
Santos, T. E. M.; Montenegro, A. A. A.; Ponciano, I. M.; Silva, J. R. L. Calibração de sonda capacitiva em três Argissolos na Bacia Representativa do Alto Ipanema, semiárido de Pernambuco. In: Congresso Brasileiro de Ciência do Solo, 32, 2009, Fortaleza. Anais... Fortaleza: SBCS, 2009b, CD-Rom

Santos, T. E. M.; Montenegro, A. A. A.; Ponciano, I. M.; Silva, J. R. L.; Silva, T. P. N.; Gusmão, M. A. M. Efeito das condições de superfície na erosão e umidade do solo, In: XVIII Simpósio Brasileiro de Recursos Hídricos, 18, 2009, Campo Grande. Anais... Campo Grande: informar instituição, 2009c. CD-Rom

SAS. The SAS System for windows: Release version: 6.8, 3.ed. Cary: 1998. CDRom.

Silva, M. A. S.; Mafra, A. L.; Albuquerque, J. A.; Bayer, C.; Mielniczuk, J. Atributos físicos do solo relacionados ao armazenamento de água em um Argissolo Vermelho sob diferentes sistemas de preparo. Ciência Rural, v.35, p.544$552,2005$.

Soil Moisture Equipment Corporation. Guelph permeameter model 2800 (User manual). Santa Barbara: Soil Moisture Equipment Corporation, 1991.27p.

Souza, E. S.; Antonino, A. C. D.; Jaramillo, R. A.; Netto, A. M.; Montenegro, S. M. G. L.; Silva, E. B. Variabilidade espacial dos parâmetros hidrodinâmicos de duas parcelas agrícolas no Estado da Paraíba. Revista Brasileira de Ciência do Solo, v.32, p.1795-1804, 2008.

Trindade, E. F. S.; Rodrigues, T. E.; Carvalho, E. J. M.; Corrêa, P. C. S. Matéria orgânica e atributos físicos de um argissolo amarelo distrófico no nordeste Paraense. Revista Amazônia: Ciência e Desenvolvimento, v.5, p.187-198, 2009.

van Genuchten, M. Th. A closed-form equation for predicting the hydraulic conductivity of unsaturated soils. Soil Science Society American Journal, v.44, p.892-898, 1980.

Vieira, S. R. Variabilidade espacial de argila, silte e atributos químicos em uma parcela experimental de um Latossolo Roxo de Campinas (SP). Bragantia, v.56, p.181-190, 1997.

Warrick, A. W.; Nielsen, D. R. Spatial variability of soil physical propertiesin the field. In: Hillel, D. (ed.). Applications of soil physics. New York: Academic Press, 1980, p.319-344.

Zhang, F. Z.; Groenevelt, P. H.; Parkin, G. W. The well-shape factor for the measurement of soil hydraulic properties using the Guelph Permeameter. Soil and Tillage Research, v.49, p.219-221, 1998. 